Thomas Linder ${ }^{1}$, Nue Milici ${ }^{1}$, Shankar Shah ${ }^{2}$

${ }^{1}$ Luzerner Kantonsspital, ${ }^{2}$ Luzerner

Kantonsspital, ENT Fellow

Learning Objectives: Competing surgical techniques and new modes of ossiculoplasties necessitate the uniform classification of cholesteatomas worldwide. We present a ChOLE staging system based on the extension, ossicular chain involvement, complications and pneumatization \& ventilation of the temporal bone.

Competing surgical techniques and new modes of ossiculoplasties necessitate the uniform classification of cholesteatomas worldwide. Whereas the pathogenesis remains a topic of debate, the extent of middle ear \& temporal bone cholesteatomas should be determined and the involvement of the ossicular chain verified. Intra- and extracranial complications are rare in well-developed countries, but challenge surgeons in more remote areas. The extent of pneumatization and ventilation of the temporal bone implicating the function of the Eustachian tube are frequently discussed, but have never been thoroughly addressed. Our ChOLE-Classification condenses $\mathrm{Ch}$ for cholesteatoma extension, $\mathrm{O}$ for ossicular chain status, L for Life threatening complications and $\mathrm{E}$ for Eustachian tube function. We present our experience with a retrospective review of 100 consecutive patients and a 9.5 years follow-up.

doi:10.1017/S0022215116003212

\section{Classification of Cholesteatoma (N743)}

\section{ID: 743.2}

First Experience with the ChOLE

Classification in Combination with a QoL questionnaire

\section{Presenting Author: Christof Röösli}

Christof Röösli ${ }^{1}$, David Baechinger ${ }^{2}$, Alex Huber ${ }^{2}$

${ }^{1}$ University Hospital Zurich, ${ }^{2}$ Department of

ENT, Head and Neck Surgery, University

Hospital Zurich, Switzerland

Learning Objectives: A questionnaire to assess HRQoL concerning the ear was developed and compared to the score of a new classification system for cholesteatoma.

Introduction: In otology, surgical outcome is most often assessed by reporting postoperative hearing thresholds. Subjective complaints are not always and systematically reported, although several patient-reported outcome measure exist for chronic otitis media. However they lack certain relevant symptoms concerning the ear a healthrelated quality of life (HRQoL).

Methods: A new questionnaire for comprehensively measuring HRQoL was developed and an electronic application was chosen to facilitate and accelerate data analysis. In a first step, it was tested in a cohort $(n=85)$ and the number of questions was reduced from 33 to 21 using sequential statistical analysis. Then the adjusted questionnaire was validated in a second cohort $(n=76)$. Finally, the validated questionnaire was tested in a cohort of patients with Otitis media cholesteatomatosa preoperatively and up to 3 months postoperatively to compare HRQoL to characteristics of the colesteatoma defined by a newly developed classification of cholesteatoma (ChOLE).

Results: Statistical analysis allowed a reduction of questions from 33 to 21. Validation revealed a Cronbach's a of 0.91 , indicating excellent internal consistency. Moreover, the questionnaire was able to discriminate between patients with chronic otitis media and healthy participants $(p<$ 0.0001 ), thus possessing good discrimination validity. Finally, first experience comparing HRQoL assessed by the questionnaire with stage of disease defined by the ChOLE classification showed good correlation.

Conclusions: Sufficient information on reliability and validity of the questionnaire was obtained. It can be applied to quantify HRQoL in patients with cholesteatoma and shows good correlations to the ChOLE classification.

\section{doi:10.1017/S0022215116003224}

Chronic Ear Diseases in developing world (R744)

\section{ID: 744.1}

\section{Developing Complex Ear Surgery in Malawi}

\section{Presenting Author: David Strachan}

\author{
David Strachan ${ }^{1}$, Wakisa Mulwafu ${ }^{2}$ \\ ${ }^{1}$ Bradford Royal Infirmary, ${ }^{2}$ Queen Elizabeth \\ Hospital, Blantyre, Malawi
}

Learning Objectives: To understand the challenges and difficulties in developing an otology service in one of the world's poorest countries. To reflect on the help that can be provided from more developed countries.

Malawi is one of the world's poorest and least developed countries. It has a population of 16 million, with over half living below the poverty line. Life expectancy is little over 50 years with 1 in 8 children dying before the age of five. The main health burden in Malawi is HIV (10\% of the population are HIV positive) along with tuberculosis and malaria, which together account for $40 \%$ of hospital deaths.

The rate of chronic ear disease is unknown due to lack of trained clinicians and difficulties in diagnosis however the population is twice as likely as those in Europe to be born with, or develop, hearing loss. Untreated ear disease is one of the causes of such loss.

The co-author is one of only two ENT surgeons in the whole country and the presenting author visited Malawi as part of a sabbatical in 2013. It was evident during this visit that whilst the infrastructure was being slowly developed there was a complete lack of expertise and equipment to carry out any complex otological surgery. With the support of various charitable organisations and associated industry the visit subsequently led to four cochlear implants being successfully implanted on 2 separate visits to Malawi.

Due to these developments the facilities are now such that future plans are in place to carry out regular weeks of otological surgery alongside developing a CI programme. These weeks will facilitate the short term aim of training Malawian (non-medical) clinical officers in 